have since reported a stream of new scientific fraud cases. These include the scientifically important and much-reported case at the Max Planck Institute for Plant Breeding in Cologne, where a technician was able to deceive the scientific world for years by fiddling a key assay, and the curious affair at the University of Giessen where a young veterinary scientist, stripped of his $\mathrm{PhD}$, has been charged with trying to kill his whistle-blower by spiking his tea with digitoxin.

But according to the authors of this fascinating, if somewhat fatalistic book, cheating in German science is not just about the present — it has a long history, and possibly a solid future ahead of it.

Their thesis, coherently argued, is that cheating is both widespread and intrinsic to science, riddled as it is with what they alarmingly refer to as "diseases of science" - the competition for research funds, the pressure to publish and the fight for recognition in Germany's rigidly hierarchical academic society.

They argue, correctly, that the German scientific community had, at least until now, kept its collective mind closed to the possibility that scientific misconduct could exist. 'Idealists' believed that science was too intrinsically pure to allow for cheating; 'rationalists' argued that science must always expose fiction because experiments are destined to be repeated; and 'nationalists' claimed that scientific fraud could never happen in Germany, where scientists have not been exposed to the same pressure as their US colleagues.

The authors efficiently dispense with these arguments. Some German scientists worked on Mengele's experiments in the Nazi era, so science is not intrinsically pure; experiments can lie dormant in the literature for years before they are repeated, if at all; science is global, so there is no such thing as a national scientific culture.

They applaud the way research organizations responded to the Herrmann and Brach affair by designing codes of good scientific practice and efficient mechanisms for handling fraud within the research institution where it occurs, and by limiting damage done to the research community at large. But they fear that the unwillingness of universities to adopt the new rules will allow fraud to continue in a new regime of complacency: "Problem erkannt - Gefahr gebannt" (problem recognized, danger eliminated).

This judges too quickly. It is true that universities and research institutes initially displayed innate hostility to guidance from above - guidance, moreover, which seemed to demand a public acknowledgement that they could, in principle, harbour cheats. But universities are already accepting that rules must be set, if only because this is now a condition for eligibility for most sources of public research funds.
Der Sündenfall's message may err on the side of alarmism, but it is certainly a good read, even though the science behind the scientific fraud is not always clearly described. It is expertly researched and its raw material has, by its very nature, a potent human element.

The book includes numerous case studies, beginning in the 1920s with Ernst Rupp, a physicist with the AEG company in Berlin, whose burning ambition to become a university academic, through fair means or foul, turned him into Germany's first known perpetrator of scientific fraud. Rupp claimed that he had carried out an untested experiment designed by Albert Einstein in 1926 to investigate the properties of light.

Showing (apparently) the interference of electron beams, he (apparently) demonstrated the particle-wave dualism of light and matter. His claim precipitated scepticism among the academic community he sought to woo, since the technological hurdles to such an experiment were, at the time, immense. Over the next few years other German physicists were able to prove that he had lied. In his defence, Rupp produced a psychiatrist's report saying that he suffered phases of "psychogenic trances combined with spiritual weakness", during which "he unconsciously published reports about physical phenomena which had the character of fiction".

It is interesting to note that in the good old days fraudsters, however bizarre their excuses, always admitted their guilt when overwhelmed by evidence. Their modern counterparts usually obey their lawyers' advice to deny it to the bitter end.

Alison Abbott is the senior European correspondent of Nature.

\section{A further string to the believers' bow}

\section{The Elegant Universe}

by Brian Greene

Jonathan Cape: 1999. 428 pp. £18.99

John Maddox

A year ago, I fell into conversation with a young woman just embarked on a PhD stint at a British university. Her thesis adviser had assigned her a project in string theory, and I asked whether she believed that string theory would indeed answer all the questions of fundamental physics. "I don't think so," she said, "but the mathematics is interesting."

Agnosticism such as this (and worse) is rife. For much of the past 15 years, almost the only rejoinder to scepticism has been the observation that Ed Witten, the Princeton theorist who has stepped into Einstein's shoes at the Institute for Advanced Study, "is a believer". But now the agnostics can read
Brian Greene's remarkable book as well.

Greene is a regular physicist at Columbia, a practitioner of string theory of distinction and a proselytizer of the cause. (He is not to be confused with his near namesake, Michael Green, who with his colleague Julian Schwartz of Caltech caused a stir in 1984 by demonstrating that strings can reconcile quantum theory and relativity.) Greene's contention is that the account given by string theory of the properties of the particles of matter is too good not to be true.

To be fair, Greene repeatedly acknowledges, although with decreasing frequency as the pages turn, that his high hopes for string theory may be disappointed. Perhaps he has shrewdly calculated that the sceptics will either have been won over by the repetition of the refrain "Strings are the cat's whiskers!", or that they will have fallen by the wayside before they reach the end which is a long way from the beginning.

Greene starts with the frank declaration that quantum mechanics and general relativity are incompatible. That, in itself, is not a radical revelation: people have been trying to 'quantize' Einstein's equations for a quarter of a century without success. Greene prefers to explain this failure qualitatively: Heisenberg's uncertainty principle requires that quantum fluctuations increase without limit as the space accessible for the specification of physical variables shrinks indefinitely. That means that space itself, which is smooth on a macroscopic scale, is microscopically far from smooth - or "differentiable", as mathematicians would say.

How does string theory resolve the difficulty? Elementary particles are no longer point-like objects, but tiny one-dimensional strings (which may be open with two loose ends or closed, like rubber bands) which, having tension, vibrate like piano strings. The energies of the normal modes of vibration then correspond to the masses of elementary particles (by the familiar rubric $E=$ $\left.m c^{2}\right)$. They are all there. Electrons, quarks and the particles that transmit the various forces - photons, the heavy bosons of the electroweak theory and the gluons that mediate the strong nuclear force. And then, magic upon magic, there are also gravitons - the massless particles of spin 2 that are supposed to be the quantum particles of the gravitational field. That is how string theory unites gravitation with the other forces.

This picture, for outsiders, is also the stumbling block to understanding. A real string could not yield the riches of the known elementary particles. External disturbance of a vibrating string, perhaps by collision with another, would change a pure vibrational state into a mixture of all others, but photons do not turn into gravitons or into quarks of different kinds. Why do events of that kind never happen? Because the strings of particle theory vibrate in 10 
dimensions - the four dimensions of relativistic space-time and six others of which we are unaware. Thus there is room for ample orthogonality to generate selection rules that prevent bizarre happenings between particles.

The most persuasive part of Greene's excellent book is that in which he persuades the reader that the problem of the six hidden dimensions is not a problem but a matter of perspective. A garden hose seen from a great distance looks like a one-dimensional object, but close up it is plainly a two-dimensional surface on which motion perpendicular to the length is perforce circular and repetitive. The length of the hose is Greene's analogy for an ordinary extended dimension; the perpendicular circular tracks stand for one of the six wrapped-up dimensions. If the radius of the circle (or of the garden hose) is small enough, it is recognizable only in close-up (which means at the highest energy).

How small are the compact radii? It seems to be agreed that the compact dimensions must be curled up with a radius in the neighbourhood of the Planck length, which is the constant with the dimensions of length formed from Planck's constant, Newton's gravitational constant and the velocity of light. Formally, the length is $\mathrm{V}\left(h G / c^{3}\right)$, where $h$ and $G$ are the quantum and gravitational constants and $c$ is the velocity of light. Numerically, the length works out at $10^{-35} \mathrm{~m}$, which is small enough for Greene's purposes.

This is the kindergarten stuff of string theory, but Greene shrinks from no obstacle in the path of understanding, instead turning each into an opportunity to make the field exciting. The doctrine of supersymmetry has become an intrinsic part of string theory - otherwise 'superstring' theory. The implication is that there are as many fundamental particles yet to be discovered as are now known, but all of them are much more massive. String theory will fall if it is ever shown that they do not exist.

The essential step forward, and the cause of Greene's rekindled enthusiasm, was taken by Witten in 1995. He showed that the five alternative string theories then defined are essentially equivalent. A weak coupling constant (or the strings' equivalent of electric charge) in one will be the equivalent of another theory with a strong coupling constant. So, says Greene, the road points ahead to the age-old dream of predicting both the contents of the Universe and the properties of those contents. Already there are people working on the notion that black holes are merely very massive elementary particles.

Even if the dream proves false, Greene has brought an absorbing field of enquiry to vivid life. The supposition that particles are not points but strings fits well with familiar phenomena. The fact that all particles of matter have intrinsic spin (which may be zero) has always provoked the question of whether spin is a property of particles or of space. The materialization of particles from apparently empty space is similarly provoking. String theory neatly answers them all.

So what lies ahead? Not even Greene is sure. String theory may not turn out to be the cat's whiskers he hopes. There are alternatives, such as Roger Penrose's twistor theory (which Greene reckons may say the same as strings). The most imaginative suggestion in this imaginative book is that the time has come to solve problems of quantum gravity in strictly quantum language and not by posing them in classical terms and then 'quantizing' them. Meanwhile, there is a whole raft of algebraic geometry to be done. The thousand and more people working in the field will need courage to do these largely thankless chores, but this splendid book will cheer them on their way.

Sir John Maddox is at 9 Pitt Street, London W8 4NX, UK.

\section{The self-centred meme}

\section{The Meme Machine}

by Susan Blackmore

Oxford University Press: 1999. 258 pp.

$£ 18.99, \$ 25$

\section{Jerry A. Coyne}

The meme, defined as "any unit of cultural transmission or any unit of imitation", was introduced by Richard Dawkins in the final chapter of The Selfish Gene. Dawkins' message was that any entity with the gene-like

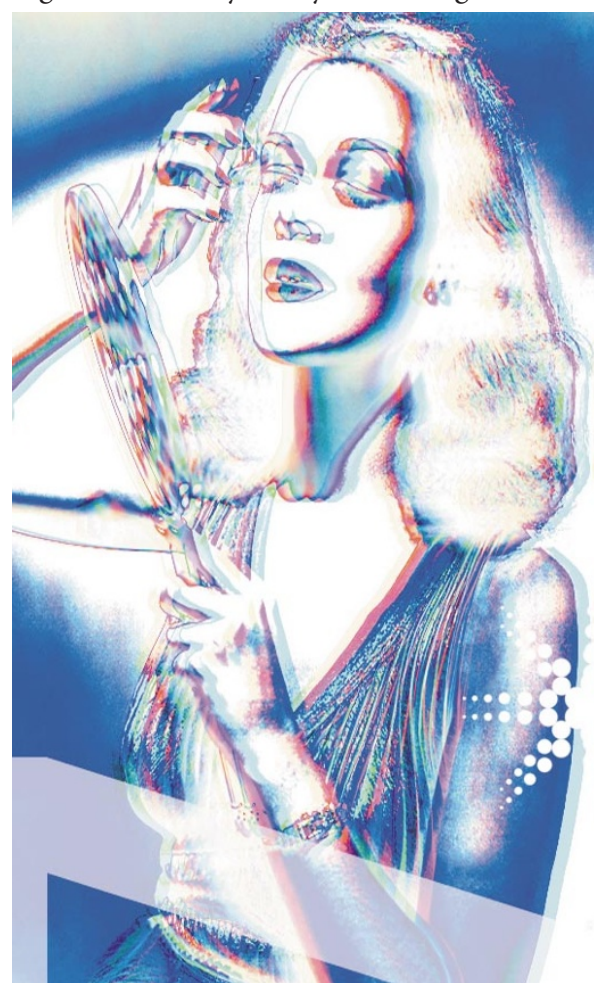

ผ๑ 1999 Macmillan Magazines Ltd properties of replication, variation and competition is a "selfish replicator" that can spread through populations by an analogue of natural selection. Memes qualify as replicators because bits of culture can be copied by imitation and compete with other units for human attention. Thus, despite the differences between memes and genes (genes, for example, are almost never passed to unrelated individuals), those memes most easily replicated and mimicked could proliferate, causing swift and important cultural change. Alarmed by the rampant and uncritical adoption of this analogy by philosophers, psychologists and the popular press, Dawkins downplayed the importance of memes in his later works.

However, in his foreword to Susan Blackmore's The Meme Machine, Dawkins notes that: "I was always open to the possibility that the meme might one day be developed into a proper hypothesis of the human mind, and I did not know how ambitious such a thesis might turn out to be." The thesis, called "memetics", has now arrived, and it is indeed ambitious. Unfortunately, Blackmore's book, aimed at both general readers and academics, proves to be a work not of science, but of extreme advocacy. Teeming with untestable speculations, indifferent to alternative theories and almost too grandiose to be taken seriously, The Meme Machine offers a convoluted and wholly unsatisfying - explanation of cultural and biological evolution.

Blackmore defines memes as "units of imitation"; her examples from humans include songs, fax machines, books, alcohol, and any device, behaviour or idea that can be copied. Memes can also be combined into

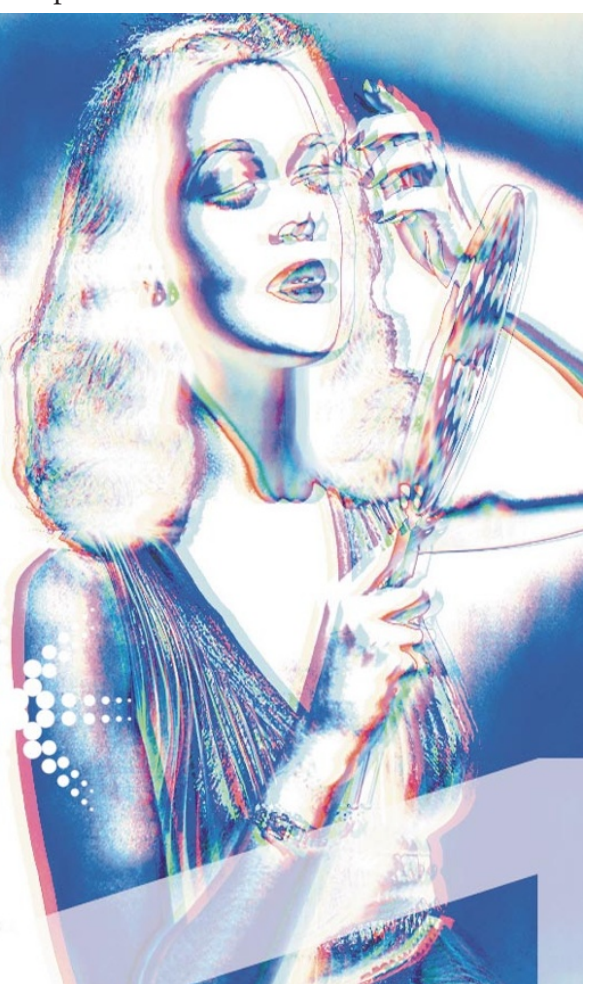

